\title{
Unusual uterine metastasis of plasmablastic lenfoma: a case report
}

\author{
Suat Karataş$^{1} \odot$, Tayfur Çift ${ }^{2} \odot$, Veysel Şal $^{1} \odot$, Meltem Tekelioğlu $^{1} \odot$, Özlem Ton $^{3} \odot$ \\ ${ }^{1}$ Department of Gynecology and Obstetrics, Şişli Hamidiye Etfal Training and Research Hospital, Istanbul, Turkey \\ ${ }^{2}$ Department of Gynecology and Obstetrics, Bursa Yüksek Ihtisas Training and Research Hospital, Bursa, Turkey \\ ${ }^{3}$ Department of Pathology, Şişli Hamidiye Etfal Training and Research Hospital, İstanbul, Turkey
}

DOI: $10.18621 /$ eurj.455365

\begin{abstract}
Plasmablastic lenfoma (PBL) is a diffuse large B-cell lymphoma (DLBCL)'s variant form that is especially reported in HIV-infected patients. PBL is a rare entity seen most commonly in the setting of immunocompromise. However, this disease may present in extraoral sites in which the genital tract appears to be the less common. Here we present a patient with uterine and fallopian mass diagnosed PBL. A 51-year-old multipara (G6P4C2) woman with a chief complaint of irregular vaginal bleeding was presented to our outpatient clinic. She had a prior history of breast cancer treated by right mastectomy and adjuvant chemotherapy.Transvaginal ultrasonography and magnetic resonance imaging both showed an uterine mass thought to be leiomyoma, which $4 \times 3 \mathrm{~cm}$ in size on the anterior wall of uterin corpus. The pathology result of the mass was found to be plasmablastic lymphoma. Genital involvement also appears to be last but possible site option to find PBL focus in immunocompetent patients.
\end{abstract}

Keywords: Plasmablastic lymphoma, uterine mass, surgery

Received: August 27, 2018; Accepted: June 1, 2019; Published Online: July 29, 2019

$\mathbf{P}$ lasmoblastic lenfoma (PBL) is a diffuse large Bcell lymphoma(DLBCL)'s variant form that is especially reported in HIV-infected patients. After time this illness is also reported in immunocompetent patients [1-3]. Despites PBL most frequently presents in oral cavity, in the literature it was reported that many other parts of the body are affected by this illness [3]. On rare occasions, female genital tract organs can be the initial site of lymphoproliferative malignancies $[4,5]$.

It generally presents earlier in life in HIV-infected patients compared with immunocompetent patients (median age, 38 years vs. 58 years, respectively). PBL has a clear male predominance, and the incidence rises in HIV-infected patients with decreased CD4 count
[1]. We present an interesting case of plasmablastic lymphoma, which is a rare type of non-Hodgkin lymphoma (NHL) that is typically diagnosed in HIV-positive patients and has an immunophenotype that overlaps with multiple myeloma [6]. NHL and it is estimated incidence of PBL accounts for approximately $5 \%$ of all HIV-positive NHL cases. Incidence of HIVnegative PBL is still unclear $[7,8]$. With regard to the management of PBL, the common treatments are chemotherapy, radiotherapy with or without surgical excision, or the combination of chemotherapy and radiotherapy. PBL has a poor prognosis and in the first year illness relapses at the rate of approximately $60 \%$ $[8,9]$.

Here we report a rare case of patient with plas-

Address for correspondence: Tayfur Çift, MD., Bursa Yüksek Ihtisas Training and Research Hospital, Department of Gynecology and Obstetrics, Bursa, Turkey

E-mail: tayfur_cift@yahoo.com 
moblastic lymphoma of uterus and fallopian tubes.

\section{CASE PRESENTATION}

A 51 year-old multipara (G6P4C2) woman with a chief complaint of irregular vaginal bleeding was presented to our outpatient clinic. She had a prior history of breast cancer treated by right mastectomy and adjuvant chemotherapy. Chronic renal failure treated first by peritoneal dialysis and eventually renal transplant. Physical examination did not show any abdominal or cervical mass. Transvaginal ultrasonography and magnetic resonance imaging (MRI) (Figure 1) both showed an uterine mass thought to be leiomyoma, which $4 \times 3 \mathrm{~cm}$ in size on the anterior wall of uterin corpus and tubular-cyctic mass sized approximately $5 \mathrm{~cm}$ on right ovary. A complete blood count revealed the following: hemoglobin, $10.8 \mathrm{~g} / \mathrm{L}$; white blood cell count, $12.15 \times 103 / \mathrm{uL}$ with a differential count of $75.9 \%$ segmented neutrophils, $20.3 \%$ lymphocytes, $2.9 \%$ monocytes, $0.4 \%$ basophils, and $0.5 \%$ eosinophils. There was no abnormality in Pap-smear results. Total abdominal hysterectomy and bilateral salphingo-oophorectomy was performed and the specimen was sent to the

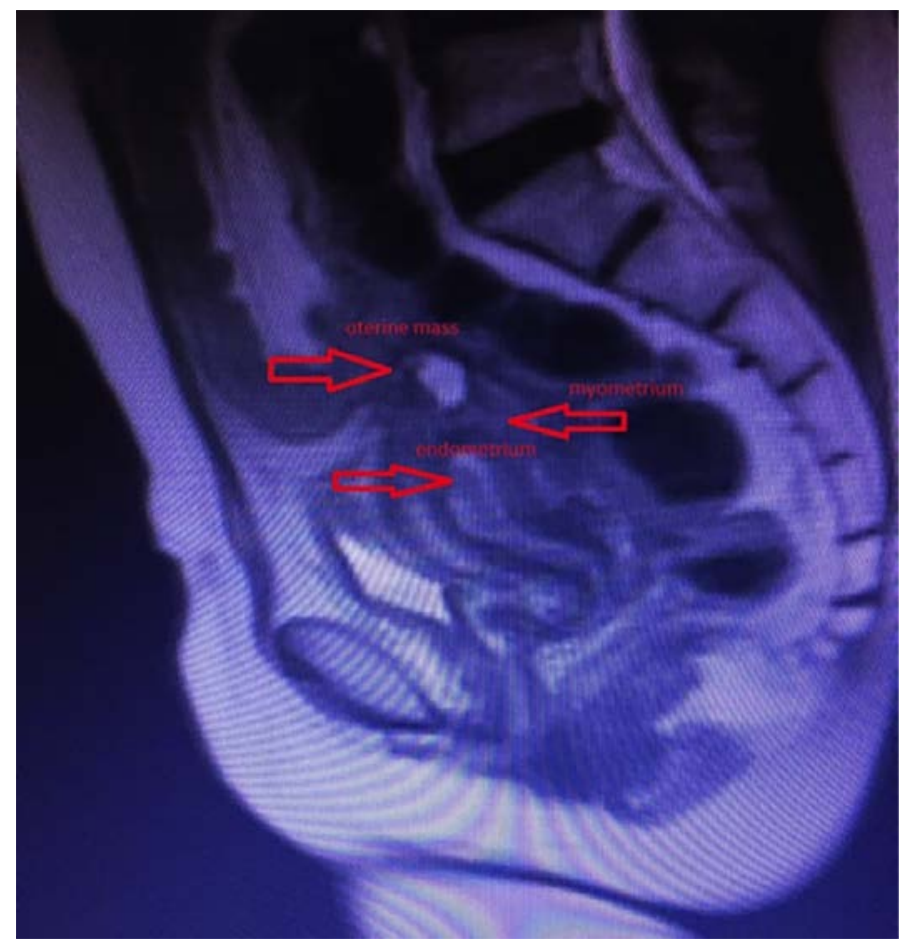

Figure 1. Magnetic resonance image of Uterine mass. Arrows shows uterine mass; endometrium and myometrium.
A
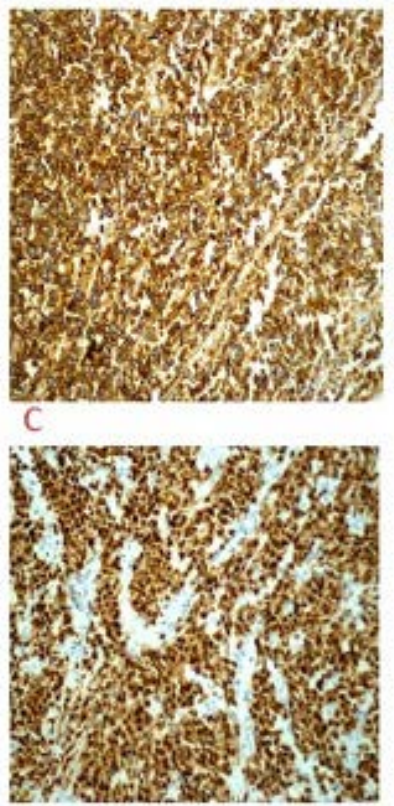

B

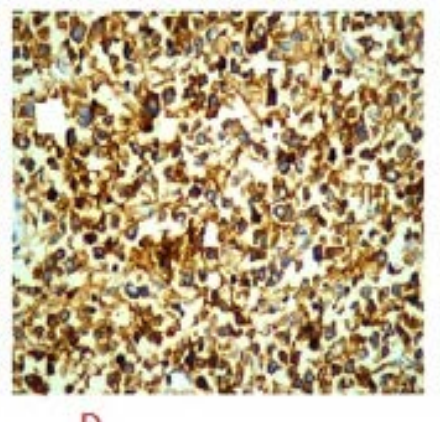

D

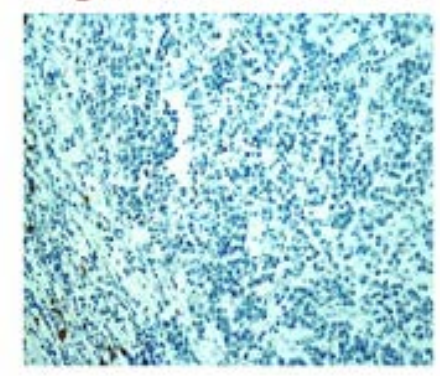

Figure 2. Immunohistochemical markers. (A) CD 38 with membranous positive $(\mathrm{CD} 38 \times 200)$, (B) CD 138 with membranous positive (CD138 $\times 200)$, (C) MUM-1 with nuclear positive (MUM-1×200), and (D) CD 20 with negative $($ CD20×200).

Department of Pathology for pathological-anatomical analysis.

On postoperative period, hemoglobin and hematocrit levels began to drop, in spite of blood transfusion. On the first day, hemoglobin was $8.9 \mathrm{~g} / \mathrm{L}$, transfusion with 2 units of erithrocyte suspension was made. However, there were no increase on levels, on the contrary, until pos-operative day 18 , hemoglobin levels continued to drop with the last value being 7.6 $\mathrm{g} / \mathrm{L}$. Transvaginal ultrasonography and computed tomography (CT) scan were performed. They showed a loculated, hyperdense lesion on operation site, sized $7.5 \times 5 \mathrm{~cm}$ thought to be an abscess or hematoma. CT scan also showed a hyperreactive lymph node sized $2.7 \times 1.5 \mathrm{~cm}$ on right inguinal region. Ongoing ampiric antibiotherapy was changed to piperacillin-tazobactam $4.5 \mathrm{~g}$ per three times a day. In the meantime, pathology report of operation specimen was completed and result came as malignant. A plasmablastic neoplasm showing lambda light chain monotype was localized diffusely on serosal surfaces of the uterus, paracervical and parametrial tissues, external surface and muscular layer of both fallopian tubes, omentum was detected. On microscopic examination, morphologically plasmablastic, multinuclear with large, pleomorphic 

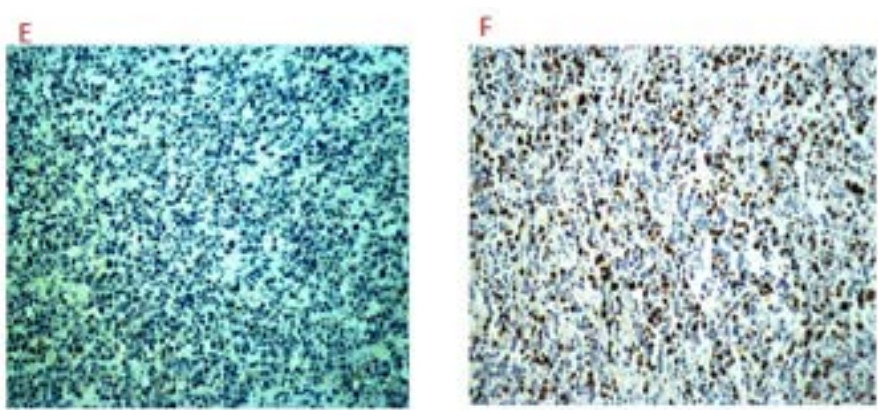

G

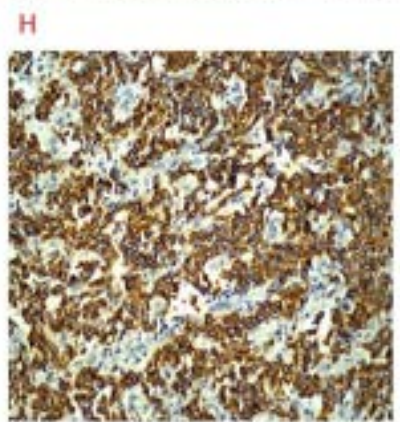

Figure 3. Immunohistochemical markers of speciment. (E) CD 79alfa with negative (CD 79alfa), (F) EBER with positive $($ EBER CISH $\times 200)$, (G) Kappa with negative (Kappa $\mathrm{CISH} \times 200$ ), and $(\mathrm{H})$ Lambda positive (Lambda CISH $\times 200$ ).

nuclei and eccentric nuclei localization cells were seen. In immunohistochemical studies, neoplastic cells were CD38(+), CD138(+), MUM-1(+), CD20(-) (Figure 2), $\mathrm{CD} 79 \mathrm{a}(-), \operatorname{EBER}(+), \operatorname{KAPPA}(-)$, LAMBDA $(+)$ (Figure 3), CD56(+), CD117(+), CD3(), $\operatorname{CD} 15(-), \operatorname{LCA}(+)$, bcl-1(-), bcl-2(-), ALK(-), pancytokeratin(-), cytokeratin 7(-), cytokeratin 20(-). Ki67 proliferation index was $80-90 \%$. Bone marrow aspiration was performed, however, the result was normocellular marrow. A lymph node biopsy was planned, tru-cut biopsy of the right inguinal lymph nodes was resulted in confirmation of the previous report, neoplastic lymphoid infiltration matching with lymphoplasmacytic lymphoma. PET CT scan showed increased FDG uptake on little curvature of the stomach and various peritoneal serosal surfaces. Antibiotic therapy ended and the patient was transferred to hematology clinic on postoperative $32^{\text {nd }}$ day.

\section{DISCUSSION}

Plasmablastic lymphoma (PBL) is a very rare and an aggressive type of lymphoma. It displays some features of multiple myeloma (MM), and some features of diffuse large B-cell lymphoma (DLBCL). This tumor was described in the oral cavity of HIVpositive patients firstly in 1997. Subsequently, the tumor has been described in regions other than the oral cavity, particularly in HIV negative patients [3]. Differences between HIV-positive and HIV-negative cases of PBL are significant. Clinically, HIV-positive patients have a stronger male predominance, early lifetime onset, and classically manifest with EBV positive oral cavity lesions. Additionally, HIV-positive patients with PBL respond to antiretroviral medications and have improved overall survival [6].

In the literature, it was mentioned that a weak male predominance (male/female ratio of 1.8) was noticed upon the PBL cases. HIV-associated PBL cases male/female ratio was reported as a 4:1. All the Chinese PBL cases included in this study were HIVnegative, suggesting HIV might be more easily affected in male patients with PBL. Actually, most PBL cases were HIV-positive. As a result of the study, the advanced clinical stage was associated with the poor survival and identified as a prognostic factor for HIV-negative PBL patients in Chinese [7].

PBL has been less frequently described in immunocompetent patients and in extraoral locations like our case. The GI tract appears to be the most common site of involvement $(20 \%)$ in HIV-negative PBL. Soft tissue (17\%), bone marrow (15\%), and skin $(12 \%)$ are the other sites that could be seen. Uterus and the fallopian tubes are the rare localizations. In chemotherapy protocols mostly used CHOP or CHOPlike regimen in PBL. Hyper-CVAD, dose-adjusted EPOCH and CODOX-M/IVAC regimen is also used in PBL. Unfortunately aggressive regimens have not shown a statistically significant improvement in outcome and survival [2, 3, 8].

HIV-negative patients was associated with worse outcomes. Despite this opinion, comparative studies have shown that HIV status does not appear to affect the outcomes in PBL. In the literature, HIV-positive patients with PBL that added antiretroviral therapy seemed to do better to chemotherapy $[6,7]$.

PBL has a characteristic immunophenotype, where in they are positive for the plasma cell markers such as CD38, CD138, and MUM1; and negative for the typical B-cell antigens such as CD20 and CD79 
$[6,9]$. As the pathological examination of our case showed that CD38, CD138 and MUM1 markers were positive and CD20 and CD79 were negative. There is usually a high level of proliferation as detected by high Ki-67 levels in PBL.6We also determined in our case that Ki67 proliferation index was $80-90 \%$. In two studies they suggested that Ki-67 values more than $80 \%$ have been seen to be an independent poor prognostic factor $[6,10]$.

In HIV-negative patients, the median overall survival was 19 months compared with 15 months in HIV-infected patients. There is no clear role for transplant in these patients. This case illustrates an interesting presentation of PBL and the challenges faced in treating these patients, because of the paucity of reported cases and the absence of a standard therapy. Exploring new therapeutic pathways by targeting activated pathologic pathways might be in the interest of these patients [1].

\section{CONCLUSION}

In conclusion, PBL is a rare entity seen most commonly in the setting of immunocompromise. The most common site involved in these patients is the oral cavity. However, this disease may present in extraoral sites in which the genital tract appears to be the less common. Genital involvement also appears to be last but possible site option to find PBL focus in immunocompetent patients. The prognosis is uniformly poor even with optimal management with cytotoxic agents.

\section{Informed consent}

Written informed consent was obtained from the patient for publication of this case report and any accompanying images.

\section{Conflict of interest}

The authors declared that there are no potential conflicts of interest with respect to the research, authorship, and/or publication of this article.

\section{REFERENCES}

[1] El Fakih R, Almahayni M, Alsermani M. Plasmablastic lymphoma presenting as exophytic skin lesions. Hematol Oncol Stem Cell Ther 2017;10:164-5.

[2] Delecluse HJ, Anagnostopoulos I, Dallenbach F, Hummel M, Marafioti T, Schneider U, et al. Plasmablastic lymphomas of the oral cavity: a new entity associated with the human immunodeficiency virus infection. Blood 1997:15;89:1413-20.

[3] Lee OJ, Kim KW, Lee GK. Epstein-Barr virus and human immunodeficiency virus-negative oral plasmablastic lymphoma. J Oral Pathol Med 2006;35:382-4.

[4] Swerdlow SH, Campo E, Pileri SA, Harris NL, Stein H, Siebert R, et al. The 2016 revision of the World Health Organization classification of lymphoid neoplasms. Blood 2016;127:2375-90.

[5] Morton LM, Wang SS, Devesa SS, Hartge P, Weisenburger DD, Linet MS. Lymphoma incidence patterns by WHO subtype in the United States, 1992-2001. Blood 2006;107:265-76.

[6] Dittus C, Sarosiek S. A case of HIV-negative plasmablastic lymphoma of the bone marrow with a unique immunophenotype. Clin Case Rep 2017;5:902-4.

[7] Han X, Duan M, Hu L, Zhou D, Zhang W. Plasmablastic lymphoma: Review of 60 Chinese cases and prognosis analysis. Medicine (Baltimore) 2017;96:e5981.

[8] Gong J, Alkan S, Anand S. A case of cutaneous plasmablastic lymphoma in HIV/AIDS with disseminated cryptococcus. Case Rep Oncol Med 2013;2013:862585.

[9] Rafaniello Raviele P, Pruneri G, Maiorano E. Plasmablastic lymphoma: a review. Oral Dis 2009;15:38-45.

[10] Komaranchath AS, Haleshappa RA, Kuntegowdenahalli LC, Kumar RV, Dasappa L, Babu G. Plasmablastic lymphoma of the gastrointestinal tract: A rare entity with a dismal prognosis. Indian J Cancer 2016;53:529-33. 